TRANSACTIONS OF THE

AMERICAN MATHEMATICAL SOCIETY

Volume 352 , Number 12, Pages 5667-5688

S 0002-9947(00)02676-3

Article electronically published on August 21, 2000

\title{
ON COBORDISM OF MANIFOLDS WITH CORNERS
}

\author{
GERD LAURES
}

\begin{abstract}
This work sets up a cobordism theory for manifolds with corners and gives an identification with the homotopy of a certain limit of Thom spectra. It thereby creates a geometrical interpretation of Adams-Novikov resolutions and lays the foundation for investigating the chromatic status of the elements so realized. As an application, Lie groups together with their left invariant framings are calculated by regarding them as corners of manifolds with interesting Chern numbers. The work also shows how elliptic cohomology can provide useful invariants for manifolds of codimension 2 .
\end{abstract}

\section{INTRODUCTION}

The study of manifolds with corners was originally developed by Cerf Cer61] and Douady Dou61 in the early 1960s, as a natural generalisation of the concept of manifolds with boundary. Its root lay in the realms of differential topology, and applications were quickly found, by Jänich [Jän68] and others, to the problem of classifying actions of transformation groups on smooth manifolds. Once Novikov's version of the Adams spectral sequence appeared in the mid 60s, it became clear that some sort of manifolds with corners also provides a geometrical framework for the realization of Adams resolutions, thereby extending Conner and Floyd's work relating the $e$-invariant and complex cobordism CF66.

During the 1970s, several authors ([AS74], Woo76], [Ste76], Kna78], Ray79, Oss82, et al.) followed the geometrical approach to stable homotopy theory by investigating the framed cobordism classes represented by various families of examples, such as Lie groups and their natural framings. This perspective opened up exciting prospects for a more geometrical analysis of Adams-Novikov phenomena, but these were never properly pursued as the emerging $B P$ machinery concentrated most minds on the power of the algebraic approach.

This paper begins the programme of unifying these two themes. It determines precisely what sort of manifolds with corners and cobordisms are needed to interpret Adams-Novikov resolutions, and lays the foundation for investigating the chromatic status of elements so realized.

Applications are given in various directions: any Lie group $G$ of rank $r$ emerges as the corner of the fibre bundle $G \times_{\mathbb{T}}\left(D^{2}\right)^{r}$. The latter has interesting Chern numbers which lead to an explicit representative of $G$ in the cobar complex. The

Received by the editors June 24, 1998.

2000 Mathematics Subject Classification. Primary 55N22, 55T15; Secondary 55Q10, 55N34, $57 \mathrm{R} 20$.

Key words and phrases. Cobordism theory, manifolds with corners, Lie groups, AdamsNovikov spectral sequence, elliptic cohomology. 
established formulae apply to any $G$ of arbitrary dimension and are illustrated by some low dimensional examples.

As another application the paper answers the question

What are the Chern numbers which determine $\beta$ at the prime 3 ?

which was raised by H.R. Miller and I.M. Singer. Needless to say, the method of the proof of 4.2.5 applies not only to $\beta$ but to any homotopy class of the sphere.

Finally, elliptic cohomology is used to define a higher version of the $e$-invariant which takes values in the group of divided congruences between modular forms. In similarity to the Todd genus of manifolds with boundaries this ' $f$-invariant' comes up as the elliptic genus of codimension 2 manifolds. In 4.3 .2 it is shown that this invariant detects the rich algebraic structure of the Adams-Novikov 2-line: a framed manifold is a corner of a $(U, f r)^{3}$-manifold iff the $f$-invariant is integral.

The author would like to thank the referee for the historical background to the subject which he incorporated into the introduction. He was not aware of [Jän68] and the notion of an $\langle n\rangle$-manifold, which turned out be the same as his earlier definition of a manifold with simplicially indexed corners. He also would like to thank Matthias Kreck, Erich Ossa and Jack Morava for their encouragement and for helpful discussions. He is especially indebted to Haynes Miller, without whom this work would not have taken place.

\section{Prerequisites}

2.1. Manifolds with corners and $\langle n\rangle$-manifolds. A differentiable manifold with corners (Dou61], Cer61], et al.) is a topological $\partial$-manifold $X$ together with a $C^{\infty}$ structure with corners. That is, $X$ is covered by charts

$$
\phi: \Omega \longrightarrow \mathbb{R}_{+}^{n}=[0, \infty)^{n}
$$

which are homeomorphisms from open sets $\Omega$ onto open subsets of $\mathbb{R}_{+}^{n}$. Two charts $\left(\phi_{i}, \Omega_{i}\right)_{i=1,2}$ are said to be compatible iff

$$
\phi_{2} \phi_{1}^{-1}: \phi_{1}\left(\Omega_{1} \cap \Omega_{2}\right) \longrightarrow \phi_{2}\left(\Omega_{1} \cap \Omega_{2}\right)
$$

is a diffeomorphism. A $C^{\infty}$-structure with corners is a maximal atlas, meaning a maximal system of compatible charts.

For any $x \in \Omega$ the number of zeros $c(x)$ in $\phi(x)$ does not depend on the choice of a chart $(\Omega, \phi)$. A boundary hypersurface or a connected face of $X$ is the closure of a component of $\{x \in X \mid c(x)=1\}$. A $k$-dimensional submanifold of $X$ is a closed subset $S$ with the property that for each $x \in S$ one can find a chart $(\Omega, \phi)$ of $X$ with respect to which $S \cap \Omega$ becomes an open subset of $\mathbb{R}_{+}^{k} \cong \mathbb{R}_{+}^{k} \times\{1\}^{n-k} \subset \mathbb{R}_{+}^{n}$.

For some purposes this concept of a manifold with corners is too general. One typically needs to know how the faces are globally pieced together. This leads to the notion of an $\langle n\rangle$-manifold which we recall from [Jän68]: a manifold with corners $X$ is a manifold with faces if each $x \in X$ belongs to $c(x)$ different connected faces. For such a manifold $X$ any disjoint union of connected faces (for short: a face) is a manifold with faces itself. An $\langle n\rangle$-manifold is a manifold with faces $X$ together with an ordered $n$-tuple $\left(\partial_{0} X, \partial_{1} X, \ldots, \partial_{n-1} X\right)$ of faces of $X$ which satisfy the following conditions:

(1) $\partial_{0} X \cup \ldots \cup \partial_{n-1} X=\partial X$, and

(2) $\partial_{i} X \cap \partial_{j} X$ is a face of $\partial_{i} X$ and of $\partial_{j} X$ for all $i \neq j$. 
We will refer to the number $n$ as the codimension of $X 11$

There is a more categorical way to describe the data of an $\langle n\rangle$-manifold which will be useful later. Let $k=\{0,1, \ldots, k-1\}$ be an ordinal number. Its partial ordering ' $\leq$ ' defines the category $\underline{k}$ which is generated by the arrows

$$
0 \rightarrow 1 \rightarrow \ldots \rightarrow k-1 \text {. }
$$

Let $\underline{2}^{n}$ be the product of $n$ copies of $\underline{2}$. Then an $\langle n\rangle$-manifold $X$ gives rise to an $n$-dimensional cubical diagram of topological spaces (for short: an $\langle n\rangle$-diagram), by which we mean a functor from $\underline{2}^{n}$ to the category $\mathcal{T}$ op: for an object $a=$ $\left(a_{0}, \ldots, a_{n-1}\right) \in \underline{2}^{n}$ let $a^{\prime}=(1,1, \ldots, 1)-a$ and set

$$
X(a) \stackrel{\text { def }}{=} \bigcap_{i \in\left\{i \mid a \leq e_{i}^{\prime}\right\}} \partial_{i} X \quad \text { for } a \neq 0^{\prime} \quad \text { and } \quad X\left(0^{\prime}\right) \stackrel{\text { def }}{=} X .
$$

Here, $\left(e_{i} ; i=0, \ldots, n-1\right)$ denotes the standard basis of $\mathbb{R}^{n}$. For a morphism $b<a$ define $X(b<a)$ as the obvious inclusion. Note that for all $a \in \underline{2}^{n}$ each $X(a)$ itself carries the structure of a $\langle k\rangle$-manifold with $k=\sum_{i} a_{i}$, since

$$
\partial X(a)=\bigcup_{b<a} X(b)
$$

In this language the product of an $\langle n\rangle$-manifold $X$ with an $\langle m\rangle$-manifold $Y$ is defined as the $\langle n+m\rangle$-manifold

$$
X \times Y \quad: \quad \underline{2}^{n+m} \cong \underline{2}^{n} \times \underline{2}^{m} \stackrel{X \times Y}{\longrightarrow} \mathcal{T} \text { op } \times \mathcal{T} \text { op } \stackrel{\times}{\longrightarrow} \mathcal{T} \text { op } .
$$

Example 2.1.1. Since a $\langle 0\rangle$-manifold is a manifold without boundary and a $\langle 1\rangle$ manifold is a manifold with boundary, we can create many examples for $\langle n\rangle$-manifolds by using the product. For instance, the model space $\mathbb{R}_{+}^{n}$ becomes an $\langle n\rangle$ manifold this way. Also, any $\langle n\rangle$-manifold $X$ can be regarded as an $\langle n+1\rangle$-manifold when it is multiplied with the $\langle 1\rangle$-manifold $[1]=(\emptyset \longrightarrow *)$, which only consists of one point.

Example 2.1.2. Let $E \rightarrow B$ be a smooth principle bundle with structure group $G$. If $X$ is an $\langle n\rangle$-manifold on which $G$ acts smoothly, then so is $E \times_{G} X$. For instance, let $G$ be a compact Lie group of rank $r$ and $\mathbb{T}$ be a maximal torus. Then $\mathbb{T}$ acts on the product $\left(D^{2}\right)^{r}$ of $r$-discs $D^{2}$ by complex multiplication. Hence, the associated fiber bundle $G \times{ }_{\mathbb{T}}\left(D^{2}\right)^{r}$ is an $\langle r\rangle$-manifold. We will come back to this example in 3.2 .3

Example 2.1.3. In Jän68 it was shown that the removal of a tubular neighbourhood of any submanifold creates a manifold of one codimension higher.

Any manifold with corners $X$ which is embedded as submanifold in $\mathbb{R}_{+}^{n} \times \mathbb{R}^{m}$ for some $m$ can be given the structure of an $\langle n\rangle$-manifold by

$$
\partial_{k} X \stackrel{\text { def }}{=} X \cap \partial_{k} \mathbb{R}_{+}^{n} \times \mathbb{R}^{m} \quad \text { for } k=0, \ldots, n-1 .
$$

Conversely, we are going to show that any $\langle n\rangle$-manifold can be embedded in a nice way.

Definition 2.1.4. A neat embedding $\iota$ of an $\langle n\rangle$-manifold $X$ is a natural transformation $X \longrightarrow \mathbb{R}_{+}^{n} \times \mathbb{R}^{m}$ for some $m$ which satisfies the following conditions.

\footnotetext{
${ }^{1}$ We allow ourselves to denote by $X$ the manifold with faces as well as the total $\langle n\rangle$-manifold, since this is unlikely to cause any confusion.
} 
(1) $\iota(a)$ is an inclusion of a submanifold for all $a \in 2^{n}$, and

(2) the intersections $X(a) \cap\left(\mathbb{R}_{+}^{n}(b) \times \mathbb{R}^{m}\right)=X(b)$ are perpendicular for all $b<a$.

Example 2.1.5. The subset of the unit sphere of vectors with only nonnegative coordinates is a neat embeddeding of a triangle as a $\langle 3\rangle$-manifold in $\mathbb{R}_{+}^{3}$. The reader may find it amusing to picture a neat embedding of a square in $\mathbb{R}_{+}^{2} \times \mathbb{R}$ as a $\langle 2\rangle$-manifold.

The concept of a collar generalizes to $\langle n\rangle$-manifolds as follows:

Lemma 2.1.6. Any $\langle n\rangle$-manifold $X$ admits an $\langle n\rangle$-diagram $C$ of embeddings

$$
C(a<b): \mathbb{R}_{+}^{n}\left(a^{\prime}\right) \times X(a) \hookrightarrow \mathbb{R}_{+}^{n}\left(b^{\prime}\right) \times X(b)
$$

with the property that $C(a<b)$ restricted to $\mathbb{R}_{+}^{n}\left(b^{\prime}\right) \times X(a)$ is the inclusion map $i d \times X(a<b)$.

Proof. For each $i=0, \ldots, n-1$ we may find a smooth vector field $V_{i}$ defined on a neighbourhood of $\partial_{i} X=X\left(e_{i}^{\prime}\right)$ in such a way that $V_{i}$ restricted to $X(a)$ points into $X(b)$ for all $a \leq e_{i}^{\prime}$ and $a<b$. Hence we obtain the required embedding $C(a<b)$ by integrating the vector fields $\left\{V_{i}\right\}_{i \in I}$ with $I=\left\{i \mid e_{i} \leq b-a\right\}$.

Proposition 2.1.7. Any compact $\langle n\rangle$-manifold admits a neat embedding in $\mathbb{R}_{+}^{n} \times$ $\mathbb{R}^{m}$ for some $m$.

Proof. Choose a collar as in 2.1.6] and an embedding of $X(0)$ in some $\mathbb{R}^{m}$. This gives an embedding of the neighbourhood $\mathbb{R}_{+}^{n} \times X(0)$ in $\mathbb{R}_{+}^{n} \times \mathbb{R}^{m}$. Now suppose $b \in \underline{2}^{n}$ is given and an embedding

$$
\iota: O(\epsilon) \stackrel{\text { def }}{=} \bigcup_{a<b}[0, \epsilon)^{n}\left(a^{\prime}\right) \times X(a) \hookrightarrow \mathbb{R}_{+}^{n} \times \mathbb{R}^{m^{\prime}}
$$

is already constructed for some $0<\epsilon<1$. Let $i$ be any differentiable injection of $X(b)$ into some $\mathbb{R}^{m^{\prime \prime}}$. Such a map can easily be provided by extending charts from open subsets to all over $X(b)$ with the help of a partition of unity and multiplying them. Then the map

$$
\iota^{\prime}:[0, \epsilon)^{n}\left(b^{\prime}\right) \times X(b) \hookrightarrow \mathbb{R}_{+}^{n} \times \mathbb{R}^{m^{\prime}} \times \mathbb{R}^{m^{\prime \prime}} ;(t, x) \mapsto(t+b, 0, i(x))
$$

already looks good for interior points $x$ of $X(b)$. Hence, if $\rho_{1}, \rho_{2}$ is a partition of unity subordinate to the covering

$$
O(\epsilon) \cup\left([0, \epsilon)^{n}\left(b^{\prime}\right) \times X(b)-O(\epsilon / 2)\right)=[0, \epsilon)^{n}\left(b^{\prime}\right) \times X(b),
$$

then the map $\rho_{1} \iota+\rho_{2} \iota^{\prime}$ is an embedding of $[0, \epsilon)^{n}\left(b^{\prime}\right) \times X(b)$ into $\mathbb{R}_{+}^{n} \times \mathbb{R}^{m^{\prime}+m^{\prime \prime}}$ and restricts to the old one on $\bigcup_{a<b}[0, \epsilon / 2)\left(a^{\prime}\right) \times X(a)$. This procedure furnishes the desired embedding of $X$.

2.2. Cobordisms of $\langle n\rangle$-manifolds. Cobordism theories of $\langle n\rangle$-manifolds have already proved useful in the classification of group actions on closed manifolds Jän68. To interpret Adams-Novikov resolutions we need to introduce cobordism relations which take the various structures on the normal bundles of each face into account. For that purpose, consider a neat embedding of a $k$-dimensional $\langle n\rangle$ manifold in some $\mathbb{R}_{+}^{n} \times \mathbb{R}^{m}$. Its normal bundle $\nu$ turns $X$ into an $\langle n\rangle$-diagram in the category $\mathcal{T} o p_{B O_{r}}$ of spaces over $B O_{r}$ with $r=n+m-k$. More precisely, for each $a \in \underline{2}^{n}$ there is a map $\nu(a): X(a) \longrightarrow B O_{r}$ which sends a point $x \in X(a)$ to the subspace of the $\left(\sum a_{i}+m\right)$-dimensional vector space $T_{x}\left(\mathbb{R}_{+}^{n}(a) \times \mathbb{R}^{m}\right)$ consisting 
of all vectors which are perpendicular to the tangent space $T_{x} X(a)$. These maps are compatible with the inclusions of faces. In order to describe orientations and other structures on $\nu$, let $A_{1}, A_{2}, \ldots$ be a sequence of $\langle n\rangle$-diagrams of fibrations over $\mathrm{BO}_{1}, \mathrm{BO}_{2}, \ldots$ respectively. Suppose further that there are natural transformations $A_{1} \rightarrow A_{2} \rightarrow \ldots$ of $\langle n\rangle$-diagrams (for short: $\langle n\rangle$-maps) which lift the usual inclusions $\mathrm{BO}_{1} \hookrightarrow \mathrm{BO}_{2} \hookrightarrow \ldots$. Under these circumstances we define

Definition 2.2.1. An $A_{r}$-structure on $\nu$ is an $\langle n\rangle$-map $\bar{\nu}_{r}: X \longrightarrow A_{r}$ in $\mathcal{T}$ op $_{B O_{r}}$. Two $A_{r}$-structures $\overline{\nu_{r}}, \overline{\nu_{r}}{ }^{\prime}$ are considered as equivalent if they are homotopic through $\langle n\rangle$-maps.

In detail such a homotopy of $\langle n\rangle$-maps looks as follows: for each $a \in \underline{2}^{n}$ there is a homotopy $h(a): X(a) \times I \longrightarrow A_{r}(a)$ from $\bar{\nu}_{r}(a)$ to $\bar{\nu}_{r}^{\prime}(a)$ which commutes with the maps to $B O_{r}$, and for all morphisms $a<b$ the equality

$$
A_{r}(a<b) h(a)=h(b)\left(X(a<b) \times i d_{I}\right)
$$

holds. An $A_{r}$-structure on $\nu$ defines an $A_{r+1}$-structure via the inclusion $\mathbb{R}^{r} \hookrightarrow \mathbb{R}^{r+1}$.

Definition 2.2.2. An $A$-structure on $X$ is an equivalence class of $A_{r}$-structures on the normal bundle, two of such being identified if they agree for some $r_{0}$ and hence for all $r \geq r_{0}$.

Lemma 2.2.3. Two neat embeddings $\iota_{1}$ and $\iota_{2}$ of an $\langle n\rangle$-manifold $X$ into $\mathbb{R}_{+}^{n} \times \mathbb{R}^{m}$ are isotopic through neat embeddings in $\mathbb{R}_{+}^{n} \times \mathbb{R}^{2 m+n}$. Hence there are canonical correspondences of $A$-structures for different embeddings.

Proof. An explicit isotopy $h$ from $\iota_{1}$ to $\iota_{2}$ is given by the formula

$$
h_{t} \stackrel{\text { def }}{=}\left((1-t) \iota_{1}+t \iota_{2}, e^{-1 /\left(t-t^{2}\right)} \iota_{1}\right) \in \mathbb{R}_{+}^{n} \times \mathbb{R}^{m} \times \mathbb{R}^{m+n}
$$

for all $t \in(0,1)$ and $h_{0}=\iota_{1}, h_{1}=\iota_{2}$.

Let $\mathcal{M}_{*}^{A}$ be the graded set of compact $\langle n\rangle$-manifolds neatly embedded in some $\mathbb{R}_{+}^{n} \times \mathbb{R}^{m}$ together with an $A$-structure on their normal bundle. For any $\langle n\rangle$-diagram $D$ in a category $\mathcal{C}$ let $\partial_{n-1} D$ be the $\langle n-1\rangle$-diagram

$$
\underline{2}^{n-1} \cong \underline{2}^{n-1} \times \underline{1} \stackrel{i d \times 0}{\longrightarrow} \underline{2}^{n-1} \times \underline{2} \cong \underline{2}^{n} \stackrel{D}{\longrightarrow} \mathcal{C}
$$

for $n \geq 1$, and let $\partial_{-1} \mathcal{C}$ be the initial functor $\emptyset \longrightarrow \mathcal{C}$. Let $I_{n} D$ be the $\langle n+1\rangle$ diagram

$$
\underline{2}^{n+1} \cong \underline{2}^{n} \times \underline{2} \stackrel{i d \times 0}{\longrightarrow} \underline{2}^{n} \times \underline{1} \cong \underline{2}^{n} \stackrel{D}{\longrightarrow} \mathcal{C}
$$

For an $\langle n\rangle$-manifold $X$ with an $A$-structure, the operator $\partial_{n-1}$ returns the back face $\partial_{n-1} X$ together with an $\partial_{n-1} A$-structure. Note that any such $X$ is the $\partial_{n^{-}}$ boundary of the $\langle n+1\rangle$-diagram $I_{n} X$, but the latter is not an $\langle n+1\rangle$-manifold unless $X$ is empty.

Definition 2.2.4. Two objects $X, Y$ of $\mathcal{M}_{*}^{A}$ are said to be cobordant if there are $W, Z \in \mathcal{M}_{*}^{I_{n} A}$ and a diffeomorphism of $\langle n\rangle$-manifolds with $A$-structures

$$
X+\partial_{n} W \cong Y+\partial_{n} Z
$$

Here, + denotes the disjoint union of manifolds. 
Example 2.2.5. In the case of codimension $n=0$ this reduces to the familiar cobordism relation. For $n=1$ an object of $\mathcal{M}_{*}^{A}$ is a manifold $X(1)$ together with an $A(1)$-structure which reduces to a given $A(0)$-structure on its boundary $X(0)$. A null cobordism of $X$ is a $\langle 2\rangle$-manifold $W$ with structure

$$
W=\left(\begin{array}{cc}
W(0,1) \longrightarrow W(1,1) \\
\uparrow & \uparrow \\
W(0,0) \longrightarrow W(1,0)
\end{array}\right) \longrightarrow\left(\begin{array}{rr}
A(0) & \longrightarrow A(1) \\
\uparrow= & \uparrow= \\
A(0) & \longrightarrow A(1)
\end{array}\right)=I A,
$$

where the bottom row $\partial_{1} W$ coincides with $X$. That is, the boundary of $W(1,1)$ decomposes into $X(1)=W(1,0)$ and a part $W(0,1)$ which admits an $A(0)$-structure as indicated in Figure 1.

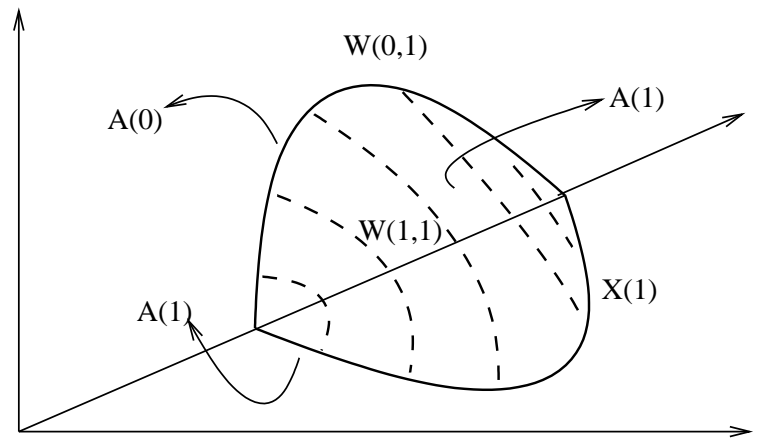

FIGURE 1.

This cobordism relation for manifolds with boundaries does coincide with the one given in [CF66] or in Sto68], p.9ff: when smoothing the corners of $W$ away we obtain a smooth manifold whose boundary is the join of two manifolds along their common boundary with appropriate structure of the normal bundle. A null bordism of the null bordism $W$, in turn, can be pictured as the region below $W$ with $A(1)$-structures on each of the three faces and compatible $A(0)$-structure on the back face.

\section{The COBORDISM GRoups}

3.1. The Pontryagin-Thom construction. As in the case of codimension $n=0$, one verifies that cobordance is an equivalence relation. Again similarly to the standard case, one shows that the equivalence classes constitute a commutative monoid $\Omega_{*}^{A}$ with unit $\emptyset$.

Proposition 3.1.1. $\Omega_{*}^{A}$ is an abelian group.

Proof. Let $\rho: I \hookrightarrow \mathbb{R}_{+}^{1} \times \mathbb{R}^{1}$ be a neat embedding of the interval $I=[0,1]$ as $\langle 1\rangle$-manifold. Then a neat embedding $\iota$ of $X$ extends to the product

$$
X \times I \stackrel{\iota \times \rho}{\hookrightarrow} \mathbb{R}_{+}^{n} \times \mathbb{R}^{m} \times \mathbb{R}_{+}^{1} \times \mathbb{R}^{1} \cong \mathbb{R}_{+}^{n+1} \times \mathbb{R}^{m+1} .
$$

The last isomorphism twists the middle coordinates. The boundary $\partial_{n}(X \times I)$ splits into two copies $X_{1}, X_{2}$ of $X$. Equip $X_{1}$ with the $A$-structure of $X$. It suffices to 
extend the $A$-structure from $X_{1}$ to an $I_{n} A$-structure on $X \times I$, since then $X_{2}$ with its induced $A$-structure is the inverse of $X$. Each face $X(a)$ of $X(b)$ admits a collar by 2.1.6] Thus $X(a<b)$ is a cofibration [Str68], and so is the inclusion of any union of faces Lil73. Since each $A_{r+1}(a) \longrightarrow B O_{r+1}$ is a fibration, we may lift the normal map as indicated in the diagram

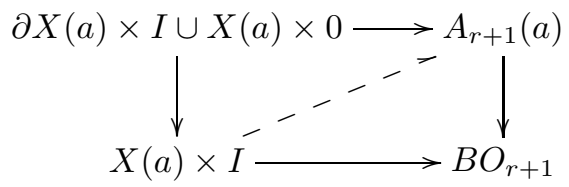

one face after another starting with the part of lowest dimension.

We will identify the abelian group $\Omega_{*}^{A}$ with the homotopy of a spectrum. For that we need a technical lemma which generalizes the desuspension theorem in stable homotopy.

For a category $\mathcal{C}$ let $\Sigma \mathcal{C}$ be the suspension category which consists of the following data: the objects of $\Sigma \mathcal{C}$ are the objects of $\mathcal{C}$ plus two more which we denote by $*$ and $*^{\prime}$; the morphisms of $\Sigma \mathcal{C}$ are the morphisms of $\mathcal{C}$, the identities of $*, *^{\prime}$ and exactly one morphism $* \rightarrow a, *^{\prime} \rightarrow a$ for any object $a$ of $\mathcal{C}$. If $\mathcal{C}$ contains an initial object $*$, let $\tilde{\mathcal{C}}$ be the full subcategory of $\mathcal{C}$ consisting of all objects except the initial. Under this condition $\mathcal{C}$ becomes a full subcategory of $\Sigma \tilde{\mathcal{C}}$ by sending $*$ to $*$.

A functor $X$ from $\mathcal{C}$ to a category $\mathcal{D}$ with initial object $*_{D}$ extends to $X^{*}: \Sigma \tilde{\mathcal{C}} \rightarrow$ $\mathcal{D}$ by the definition $X^{*}\left(*^{\prime}\right)=*_{D}$. Dually, let $\Sigma^{o p} \mathcal{C}$ be the category $\left(\Sigma\left(\mathcal{C}^{o p}\right)\right)^{o p}$ and $X_{*}: \Sigma^{o p} \widetilde{\mathcal{C}}^{o p}{ }^{o p} \rightarrow \mathcal{D}$ the extension $X\left(*^{\prime}\right)=*_{D}$ if $*_{D}$ is the terminal object of $\mathcal{D}$.

Lemma 3.1.2. Let $X$ be an $\langle n\rangle$-spectrum. Then the spectrum $\Sigma^{n}$ holim $X^{*}$ is naturally homotopy equivalent to hocolim $X_{*}$ in the category of spectra $\mathcal{S}$.

Since hocolim $X_{*}$ is the homotopy cofiber of

$$
\operatorname{hocolim}_{a \neq 0^{\prime}} X(a) \longrightarrow X\left(0^{\prime}\right)
$$

it will be denoted by $(X, \partial X)$ in the sequel. Before giving the proof of the lemma we observe that the considered homotopy (co)limit is the outcome of successive (co)fibers.

Sublemma 3.1.3. Let $\bar{\partial}_{n-1} X$ be the composite of

$$
\underline{2}^{n-1} \cong \underline{2}^{n-1} \times \underline{1} \stackrel{i d \times 1}{\longrightarrow} \underline{2}^{n-1} \times \underline{2} \cong \underline{2}^{n}
$$

with $X$. Then we have a homotopy equivalence

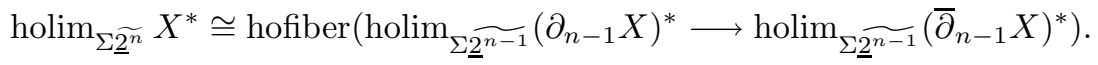

Dually, hocolim $X_{*}$ is homotopy equivalent to the homotopy cofiber of

$$
\operatorname{hocolim}\left(\partial_{n-1} X\right)_{*} \longrightarrow \operatorname{hocolim}\left(\bar{\partial}_{n-1} X\right)_{*} \text {. }
$$

Proof. The right side simply rearranges the homotopy (co)limit of the left side.

Proof of 3.1.2: The case $n=1$ is the well known fact that the suspension of the homotopy fiber gives the homotopy cofiber. Now assume the equivalence is true for 
some $n-1 \geq 0$. Then the sublemma yields

$$
\begin{aligned}
& \operatorname{holim} X^{*} \cong \operatorname{hofiber}\left(\operatorname{holim}\left(\partial_{n-1} X\right)^{*} \longrightarrow \operatorname{holim}\left(\bar{\partial}_{n-1} X\right)^{*}\right) \\
& \quad \cong \Sigma^{-1} \operatorname{hocofiber}\left(\Sigma^{-n+1} \operatorname{hocolim}\left(\partial_{n-1} X\right)_{*} \longrightarrow \Sigma^{-n+1} \operatorname{hocolim}\left(\bar{\partial}_{n-1} X\right)_{*}\right) \\
& \quad \cong \Sigma^{-n} \operatorname{hocolim} X_{*}
\end{aligned}
$$

Let $\left(\mathbb{R}_{+}^{n}\right)^{+}$denote the one-point compactification of $\mathbb{R}_{+}^{n}$ and let $X$ be an $\langle n\rangle$ diagram of pointed spaces. Then the subspace

$$
\langle n\rangle-\mathcal{T} o p^{+}\left(\left(\mathbb{R}_{+}^{n}\right)^{+}, X\right) \subset \prod_{a \in \underline{2}^{n}} \mathcal{T} o p^{+}\left(\mathbb{R}_{+}^{n}(a)^{+}, X(a)\right)
$$

consisting of all $\langle n\rangle$-maps is itself a pointed space.

Lemma 3.1.4. The pointed space holim $X^{*}$ is naturally homotopy equivalent to $\langle n\rangle-\mathcal{T} o p^{+}\left(\left(\mathbb{R}_{+}^{n}\right)^{+}, X\right)$.

Proof. We first give an another description of $\langle n\rangle-\mathcal{T} o p^{+}\left(\left(\mathbb{R}_{+}^{n}\right)^{+}, X\right)$ in terms of limits: let $\mathcal{P}_{n}$ be the full subcategory of $\left(\underline{2}^{n}\right)^{o p} \times \underline{2}^{n}$ consisting of pairs $(a, b)$ with $a \leq b$. Define a functor $R X$ from $\mathcal{P}_{n}$ to $\mathcal{T}_{o p}{ }^{+}$by

$$
R X(a, b) \stackrel{\text { def }}{=} \mathcal{T}_{o p^{+}}\left(\mathbb{R}_{+}^{n}(a)^{+}, X(b)\right),
$$

and let $R X((a, b) \rightarrow(c, d))$ be the map

$$
f \mapsto\left(\mathbb{R}_{+}^{n}(c)^{+} \stackrel{\mathbb{R}_{+}^{n}(c \leq a)^{+}}{\longrightarrow} \mathbb{R}_{+}^{n}(a)^{+} \stackrel{f}{\longrightarrow} X(b) \stackrel{X(b \leq d)}{\longrightarrow} X(d)\right) .
$$

Then it is easy to see that $\lim _{P_{n}} R X$ coincides with $\langle n\rangle-\mathcal{T} o p^{+}\left(\left(\mathbb{R}_{+}^{n}\right)^{+}, X\right)$.

Next assume that the lemma is true for all $\langle n-1\rangle$-pointed spaces and $n-1 \geq 0$. Write $e v_{0}$ for the evaluation-at-0 map. Since the previous sublemma also holds for pointed spaces instead of spectra, we get by induction

$$
\begin{aligned}
& \operatorname{holim} X^{*} \cong \operatorname{hofiber}\left(\lim R \partial_{n-1} X \longrightarrow \lim R \bar{\partial}_{n-1} X\right) \\
& \cong \lim \left(\lim _{\mathcal{P}_{n-1}} \mathcal{T} \text { op }^{+}\left(\mathbb{R}_{n}^{+}\left(e_{n-1}\right), R \bar{\partial}_{n-1} X\right) \stackrel{e v_{0}}{\longrightarrow} \lim _{\mathcal{P}_{n-1}} R \bar{\partial}_{n-1} X \leftarrow \lim _{\mathcal{P}_{n-1}} R \partial_{n-1} X\right) \\
& \cong \lim _{(a, b) \in \mathcal{P}_{n-1}} \lim \left(\mathcal{T} o p^{+}\left(\left(\mathbb{R}_{+}^{n}\right)^{+}\left(a+e_{n-1}\right), X\left(b+e_{n-1}\right)\right)\right. \\
& \left.\stackrel{e v_{0}}{\longrightarrow} \mathcal{T}_{o p^{+}}\left(\left(\mathbb{R}_{+}^{n}\right)^{+}(a), X\left(b+e_{n-1}\right)\right) \leftarrow \mathcal{T}_{o p^{+}}\left(\left(\mathbb{R}_{+}^{n}\right)^{+}(a), X(b)\right)\right) \\
& \cong \lim _{\mathcal{P}_{n}} R X \cong\langle n\rangle-\mathcal{T} \text { op }^{+}\left(\mathbb{R}_{+}^{n}, X\right) .
\end{aligned}
$$

The second equivalence uses the fact that $e v_{0}$ is a fibration with contractible total space. The third one is a consequence of the exponential law and a rearrangement of the limit.

Before coming to the main result of this section, some words about the category of $\langle n\rangle$-spectra are necessary. The category of spectra $\mathcal{S}$ over a fixed universe is enriched over $\mathcal{T} o p^{+}$. That is, the morphism sets are pointed topological spaces (see Elm88]). Hence, the category $\langle n\rangle-\mathcal{S}$ consisting of strictly commuting $\langle n\rangle$-diagrams in $\mathcal{S}$ can also be enriched over $\mathcal{T} o p^{+}$as in (3.1.1).

Theorem 3.1.5. Let $M A$ be the $\langle n\rangle$-diagram of Thom spectra of $A$. Then the Pontryagin-Thom construction gives isomorphisms of groups

$$
\Omega_{*}^{A} \cong \pi_{*}(M A, \partial M A) \cong \pi_{*}\left(\langle n\rangle-\mathcal{S}\left(\left(\mathbb{R}_{+}^{n}\right)^{+}, M A\right)\right) .
$$


Proof. First note that the previous lemmas give isomorphisms of groups for all $k \geq 0$ :

$$
\begin{aligned}
& \pi_{k}(M A, \partial M A) \cong \pi_{k-n}\left(\operatorname{holim} M A^{*}\right) \cong \operatorname{colim}_{r} \pi_{k-n+r}\left(\operatorname{holim} M A_{r}^{*}\right) \\
& \quad \cong \operatorname{colim}_{r} \pi_{k-n+r}\left(\langle n\rangle-\mathcal{T} o p^{+}\left(\left(\mathbb{R}_{+}^{n}\right)^{+}, M A_{r}\right)\right) \cong \pi_{k-n}\left(\langle n\rangle-\mathcal{S}\left(\left(\mathbb{R}_{+}^{n}\right)^{+}, M A\right)\right) .
\end{aligned}
$$

Next suppose $X$ is a $k$-dimensional $\langle n\rangle$-manifold with an $A$-structure which is nicely embedded in $\mathbb{R}_{+}^{n} \times \mathbb{R}^{m}$. Then its normal bundle $\nu$ lies in $\mathbb{R}_{+}^{n} \times \mathbb{R}^{m} \times \mathbb{R}^{n+m}$. The set $\nu \leq \epsilon$ of normal vectors of length smaller than some $\epsilon>0$ are mapped under the addition map $(a, b) \mapsto a+b$ to $\mathbb{R}_{+}^{n} \times \mathbb{R}^{m}$. In fact, if $\epsilon$ is assumed to be small enough, this gives an inclusion $\nu^{\leq \epsilon} \hookrightarrow \mathbb{R}_{+}^{n} \times \mathbb{R}^{m}$. Hence the collapse map $\left(\mathbb{R}_{+}^{n}\right)^{+} \wedge S^{m} \cong\left(\mathbb{R}_{+}^{n} \times \mathbb{R}^{m}\right)^{+} \longrightarrow \nu^{\leq \epsilon} / \nu^{=\epsilon}$ is well defined. When multiplying with $1 / \epsilon$ we may compose this map with the structure map $M \nu \rightarrow M A_{r}$ for $r=n+m-k$. Since each face $\mathbb{R}_{+}^{n}(a)^{+} \wedge S^{m}$ is sent to $M A_{r}(a)$, we obtain an object $\theta(X)$ in $\pi_{k-n+r}\left(\langle n\rangle-\mathcal{T} o p^{+}\left(\left(\mathbb{R}_{+}^{n}\right)^{+}, M A_{r}\right)\right)$. An $I_{n} A$-manifold

$$
W \hookrightarrow \mathbb{R}_{+}^{n+1} \times \mathbb{R}^{m} \cong \mathbb{R}_{+}^{n} \times \mathbb{R}^{m} \times \mathbb{R}_{+}^{1}
$$

defines a null homotopy $\left(\mathbb{R}_{+}^{n}\right)^{+} \wedge S^{m} \wedge \mathbb{R}_{1}^{+} \longrightarrow M A$ under this construction. Similarly, taking an isotopy shows that the class of $\theta$ is independent of the embedding of $X$. Hence, $\theta$ induces a cobordism invariant $\Omega_{k}^{A} \longrightarrow \pi_{k}(M A, \partial M A)$ which is easily verified to be a homomorphism. We next show that $\theta$ is epic. Let

$$
g:\left(\mathbb{R}_{+}^{n}\right)^{+} \wedge S^{m} \longrightarrow M A_{r}
$$

be an $\langle n\rangle$-map and let $M O_{r, s}$ be the Thom space of the canonical bundle over the Grassmannian space $G_{r, s}$ of $r$-dimensional subvector spaces in $\mathbb{R}^{s}$. Then the composite of $g$ with the projection to $M O_{r}$ already maps to $M O_{r, s}$ for some $s$. Call this map $h$. Since locally the inclusion $G_{r, s} \subset\left(M O_{r, s}-\infty\right)$ looks like $\mathbb{R}^{d} \subset \mathbb{R}^{l}$, the restriction of h to $U=h^{-1}\left(\mathbb{R}^{l}\right) \subset \mathbb{R}_{+}^{n} \times \mathbb{R}^{m}$ can be extended to an open subset of $\mathbb{R}^{n+m}$. Hence the usual local approximation theorem applies, and we may assume that $h$ is differentiable on $h^{-1}\left(M O_{r, s}-\infty\right)$. The regular values of $h$ in $\mathbb{R}^{l} / \mathbb{R}^{d}$ are dense even when $h$ is restricted to $U \cap\left(\mathbb{R}_{+}^{n}(a)-\partial \mathbb{R}_{+}^{n}(a)\right) \times \mathbb{R}^{m}$ for each $a \in \underline{2}^{n}$. Let $y_{j}$ be a null sequence in $\mathbb{R}^{l}$ such that $y_{j} \bmod \mathbb{R}^{d}$ are regular values of $h$ for all $a \in \underline{2}^{n}$. Then the sequence $h_{j}=h-y_{j}$ shows that $h$ may be deformed to be transverse to $G_{r, s}$ for all $a$. Now the preimage $X=h^{-1} G_{r, s}$ is an $\langle n\rangle$-manifold. The transversal embedding of $X$ in $\mathbb{R}_{+}^{n} \times \mathbb{R}^{m}$ can again be deformed to satisfy the orthogonality condition with the help of 2.1.7. The obvious $A$-structure of $X$ completes the construction of the preimage of $g$. The injectivity is shown in a similar fashion.

For $n=0$ the theorem reduces to the original Thom isomorphism [Tho54] based on $A$-bordism. In the case $n=1$ and $A=(E U \longrightarrow B U)$ we obtain the $(U, f r)$ bordism of Conner and Floyd [CF66]: the pair $(M A, \partial M A)$ is the homotopy cofiber $\Sigma \overline{M U}$ of the unit $S^{0} \longrightarrow M U$. Moreover, when we define $A^{s}$ to be the $s$-fold product of the $\langle 1\rangle$-diagram $(E U \longrightarrow B U)$ as in (2.1.1) and set $\Omega_{*}^{A^{s}}=\Omega_{*}^{(U, f r)^{s}}$, then we have

Corollary 3.1.6. $\Omega_{k}^{(U, f r)^{s}} \cong \pi_{k-s}\left(\bigwedge^{s} \overline{M U}\right)$.

Proof. The homotopy equivalence $\left(M A^{s}, \partial M A^{s}\right) \cong \bigwedge^{s} \Sigma \overline{M U}$ is easily obtained by induction with the help of 3.1 .3 , 
The geometric interpretation of $\pi_{*}(M A, \partial M A)$ generalizes to the homology and cohomology groups of pairs of spaces $(Y, X), X \subset Y$ closed, in the standard way: an element of $\Omega_{k}^{A}(Y, X)$ is represented by a $k$-dimensional singular $I_{n} A$-manifold in $Y$ with $\partial_{n}$-boundary in $X$. Note that an $I_{n} A$-manifold with empty $\partial_{n}$-boundary provides the same data as an $A$-manifold. For smooth manifolds $Y \subset \mathbb{R}^{N}$ without boundary the elements of $\Omega_{A}^{k}(Y, X)$ can be interpreted as proper $A$-oriented maps $f: Z \longrightarrow Y$ with image in $Y-X$. That is, $Z$ is an $\langle n\rangle$-manifold nicely embedded in some $\mathbb{R}_{+}^{n} \times \mathbb{R}^{m}$, and the stable normal $\langle n\rangle$-bundle of

$$
f \times \iota: Z \longrightarrow Y \times \mathbb{R}_{+}^{n} \times \mathbb{R}^{m}
$$

is $A$-structured (see Qui71 or Dol78 for the codimension 0 case).

3.2. Adams-Novikov filtrations. In the last paragraph we saw that an $\langle n\rangle$ diagram $A$ of structure spaces over $B O$ gives rise to a cobordism theory $\Omega_{*}^{A}$ which can be identified with the homotopy of the spectrum $(M A, \partial M A)$. To analyse the latter we are going to set up a filtration.

Definition 3.2.1. A resolution of $A$ is a sequence $\left(A=A^{n}, A^{n+1}, A^{n+2}, \ldots\right)$ with

(1) $A^{s}$ is an $\langle s\rangle$-diagram of structure spaces over $B O$, and

(2) $\partial_{s-1} A^{s}=A^{s-1}$ for all $s \geq n$.

Of course, any resolution of $A=A^{n}$ gives rise to a resolution of $A^{n+k}$ for all $k \geq 0$ and for $\partial_{n-k} \cdots \partial_{n-1} A=A^{n-k}$ in the obvious way. A resolution defines a filtration by

$$
F^{s, k+s} \stackrel{\text { def }}{=} \operatorname{im}\left(\partial_{n+s-1} \partial_{n+s-2} \cdots \partial_{n}: \Omega_{k+s}^{A^{n+s}} \longrightarrow \Omega_{k}^{A}\right) .
$$

Example 3.2.2. The $\langle 1\rangle$-diagram $A^{1}=(E U \rightarrow B U)$ can be resolved by the sequence $A^{k}=\left(A^{1}\right)^{k}$ for $k=1,2, \ldots$ The associated filtration of the framed bordism groups $\Omega_{*}^{f r} \cong \pi_{*}^{s t}$ coincides with the Adams-Novikov filtration: the map

$$
\Omega_{k+1}^{(U, f r)^{s+1}} \cong \pi_{k-s}\left(\left(\bigwedge^{s} \overline{M U}\right) \wedge \overline{M U}\right) \longrightarrow \pi_{k-s}\left(\bigwedge^{s} \overline{M U} \wedge S^{0}\right) \cong \Omega_{k}^{(U, f r)^{s}}
$$

is the boundary map $\partial_{s}$.

We stay for a while with the classical Adams-Novikov filtration based on $M U$ and draw some more corollaries of our theorem. Let $G$ be a compact, connected Lie group. Its tangent bundle can be trivialized by choosing a basis of its Lie algebra $\mathfrak{g}$ and using left multiplication to translate the basis to the other tangent spaces. This trivialization depends only on the choice of an orientation of $\mathfrak{g}$, and is usually called the left invariant framing $\mathcal{L}$ of $G$. Hence, any oriented and compact $G$ gives rise to an element of the framed bordism group. The following result is due to Atiyah and Smith for $r \leq 2$ and to Knapp for the general case.

Corollary 3.2.3. AS74 Kna78] Any compact, connected and oriented Lie group of rank $r$ has filtration $r$.

Proof. Choose a maximal torus $\mathbb{T}$ and an isomorphism of $\mathbb{T}$ with a product of circles $\left(S^{1}\right)^{r}$. Then its Lie algebra $\mathfrak{t} \cong \mathbb{R}^{r}$ is contained in $\mathfrak{g}$, and we may assume that the left invariant framing of $G$ comes from an extension of the standard basis of $\mathbb{R}^{r}$ to a basis of $\mathfrak{g}$. Hence, if $G$ is considered as a right principal bundle $p$ over the homogeneous space $G / \mathbb{T}$ of left cosets, then the framing of the tangents along the 
fibre $G \times_{\mathbb{T}} T\left(S^{1}\right)^{r} \cong G \times \mathbb{R}^{r}$ coincides with the left translation of the basis of $\mathfrak{t}$, and there is a splitting of trivialized bundles

$$
T G \cong p^{*}(T(G / \mathbb{T})) \oplus G \times_{\mathbb{T}} T\left(S^{1}\right)^{r} .
$$

Next extend the action of $\mathbb{T}$ on $\left(S^{1}\right)^{r}$ to the product $\left(D^{2}\right)^{r} \subset \mathbb{C}^{r}$ in the standard way. Since $\left(D^{2}\right)^{r}$ is $\mathbb{T}$-equivariantly contractible, the bundle along the fibres of $G \times_{\mathbb{T}}\left(D^{2}\right)^{r}$ is isomorphic to the sum of complex line bundles

$$
p^{*}\left(G \times_{\mathbb{T}} \mathbb{C}^{r}\right)=l_{0} \oplus l_{1} \oplus \cdots \oplus l_{r-1} .
$$

Thus the splitting

$$
\begin{aligned}
T\left(G \times_{\mathbb{T}}\left(D^{2}\right)^{r}\right) & \cong p^{*}(T(G / \mathbb{T})) \oplus G \times \times_{\mathbb{T}} T\left(D^{2}\right)^{r} \\
& \cong p^{*}\left(T(G / \mathbb{T}) \oplus l_{0} \cdots \oplus l_{r-1}\right.
\end{aligned}
$$

defines a $U^{r}$-structure which is compatible with the framings on the faces. Summarizing, we gave a $(U, f r)^{r}$-structure to the $\langle r\rangle$-manifold $G \times \times_{\mathbb{T}}\left(D^{2}\right)^{r}$ in such a way that its corner is the framed manifold $G$. Thus the result follows from 3.1.6 and 3.2 .2 .

The left invariant framing of $G$ can be twisted by elements $\alpha$ of $\widetilde{K O}^{-1} G$ as follows: since $G$ is compact, the element $\alpha$ gives rise to an automorphism $\tilde{\alpha}$ of the trivial bundle $G \times \mathbb{R}^{N}$ for some $N$. Choosing $N$ large enough, we may embed $G$ into $\mathbb{R}^{k+N}$ with trivialized normal bundle and compose the framing of $\nu$ with $\tilde{\alpha}$. The resulting reframed $G$ will be denoted by $\left[G, \mathcal{L}^{\alpha}\right]$.

Corollary 3.2.4 ([Kna78]). $\left[G, \mathcal{L}^{\alpha}\right]$ has filtration $i$ if $\alpha$ lies in the image of the map $p^{*}: \widetilde{K O}^{-1}\left(G / \mathbb{T}^{i}\right) \longrightarrow \widetilde{K O}^{-1} G$ for some subtorus $\mathbb{T}^{i} \subset \mathbb{T}$ of $G$.

Proof. First note that (3.2.1) really is a $\mathbb{T}^{i}$-equivariant splitting of the left invariantly trivialized tangent bundle into trivialized bundles. Hence, for a faithful representation $G \subset O(n) \subset \mathbb{R}^{n^{2}}$ the normal bundle will be $\mathbb{T}^{i}$-equivariantly trivialized if $n$ is large enough. Moreover, the automorphism $\tilde{\alpha}$ acts on the trivial bundle $G \times R^{N}$ for $N=n^{2}-k$ in a $\mathbb{T}^{i}$-equivariant fashion by assumption. Thus there is a $\mathbb{T}^{i}$-equivariant trivialization of the reframed $T G \oplus G \times \mathbb{R}^{N}$. When dividing out the action and framing $G \times_{\mathbb{T}^{i}}\left(T S^{1}\right)^{i}$ as usual, we end up with a new framing of $G / \mathbb{T}^{i}$ which makes

$$
T G \oplus G \times \mathbb{R}^{N} \cong p^{*}\left(T G / \mathbb{T}^{i}\right) \oplus G \times_{\mathbb{T}^{i}} T\left(S^{1}\right)^{i} \oplus G \times \mathbb{R}^{N}
$$

into an isomorphism of framed bundles. Now we may proceed as in 3.2.3.

The classical Adams-Novikov spectral sequence generalizes to arbitrary resolutions $\left(A^{s}\right)_{s=0,1, \ldots}$ as follows: For any $\langle s+1\rangle$-diagram $A^{s+1}$ let $Z A^{s+1}$ be the $\langle s+1\rangle$-diagram $\bar{\partial}_{s} A^{s+1} \times[1]$, where $\bar{\partial}_{s}$ was defined in [3.1.3] and [1] is the $\langle 1\rangle$ diagram $(\emptyset \longrightarrow *)$ as in 2.1 .2 . Then an $\langle s\rangle$-manifold $X$ with $A^{s}$-structure gives rise to the $Z A^{s+1}$-manifold $j(X)=X \times[1]$, since the normal bundle of $X \times[1]$ comes with a structure map to

$$
A^{s} \times[1]=\partial_{s} A^{s+1} \times[1] \longrightarrow \bar{\partial}_{s} A^{s+1} \times[1]=Z A^{s+1} .
$$

Furthermore, any $Z A^{s}$-manifold $Y$ may be viewed as $A^{s}$-manifold $k(Y)$ in the obvious way. 
Proposition 3.2.5. The triangle

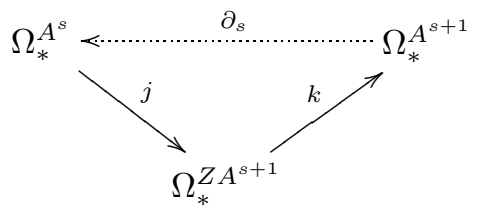

defines an exact couple. The associated first quadrant spectral sequence with $E_{1}^{s, t}$ term $\Omega_{t}^{Z A^{s+n}}$ for $s, t \geq 0$ admits a monomorphism

$$
0 \longrightarrow F^{s, t} / F^{s+1, t+1} \longrightarrow E_{\infty}^{s, t} \text {. }
$$

Proof. It is not hard to show the exactness of the triangle in pure geometrical terms. However, since we have 3.1 .5 and 3.1 .3 available, we only need to observe that

$$
\begin{aligned}
& \operatorname{hocofiber}\left(\operatorname{hocolim}\left(M A^{s}\right)_{*} \stackrel{j}{\longrightarrow} \operatorname{hocolim}\left(M Z A^{s+1}\right)_{*}\right) \\
& \quad \cong \operatorname{hocofiber}\left(\operatorname{hocolim}\left(\partial_{s} M A^{s+1}\right)_{*} \longrightarrow \operatorname{hocolim}\left(\overline{\partial_{s}} M A^{s+1}\right)_{*}\right) \\
& \cong \operatorname{hocolim}\left(M A^{s+1}\right)_{*} .
\end{aligned}
$$

The second statement is clear.

Example 3.2.6. Let $B$ be a $\langle 1\rangle$-diagram of structure spaces with contractible $B(0)$. Then $A \times B(0)$ can be resolved by the sequence $A^{s}=A \times B^{s}$, and

$$
\Omega_{*}^{Z A^{s}} \cong M B(1)_{*}\left((M A, \partial M A) \wedge \bigwedge^{s} \Sigma \overline{M B(1)}\right)
$$

is a $\pi_{*} M B(1)$-module. In this case the proposition is a geometric description of the Adams-Novikov spectral sequence Ada74 for $(M A, \partial M A)$ based on $M B(1)$.

\section{INVARIANTS OF $\langle n\rangle$-MANIFOLDS}

4.1. Genera of $\langle n\rangle$-manifolds and $E_{2}$-invariants. The homotopy category of $\langle n\rangle$-spectra is symmetric monoidal: the product of $\langle n\rangle$-spectra $E$ and $F$ is defined by the composite

$$
\underline{2}^{n} \stackrel{\Delta}{\longrightarrow} \underline{2}^{n} \times \underline{2}^{n} \stackrel{E \times F}{\longrightarrow} \mathcal{S} \times \mathcal{S} \stackrel{\wedge}{\longrightarrow} \mathcal{S} .
$$

In detail, let $L$ denote the isometry operad as defined in [LMS80] and set

$$
(X \wedge Y)(a \leq b) \stackrel{\text { def }}{=} L(2) \rtimes(X(a \leq b) \wedge Y(a \leq b)) .
$$

Then $X \wedge Y$ gives a new strictly commutative $\langle n\rangle$-diagram in $\mathcal{S}$. Hence, it makes sense to talk about $\langle n\rangle$-ring spectra and ring maps between them.

Definition 4.1.1. An A-orientation of an $\langle n\rangle$-ring spectrum $E$ is a ring map $g: M A \longrightarrow E$. Any such gives rise to a genus of $A$-manifolds, by which we mean the associated map in homotopy

$$
g_{*}: \Omega_{*}^{A} \cong \pi_{*}(M A, \partial M A) \longrightarrow \pi_{*}(E, \partial E) .
$$

If $E$ is $A$-oriented, then any compact $k$-dimensional $A$-manifold $X$ admits a fundamental class

$$
[X]_{E} \stackrel{\text { def }}{=} g_{*}(X \stackrel{i d}{\longrightarrow} X) \in(E, \partial E)_{k}(X)=\pi_{k}\left(X_{+} \wedge(E, \partial E)\right)
$$

and its dual class

$$
1_{X}^{E} \stackrel{\text { def }}{=} g_{*}(X \stackrel{\tilde{i d}}{\longrightarrow}(X-\partial X)) \in(E, \partial E)^{0}(X, \partial X)=H o-\mathcal{S}((X, \partial X),(E, \partial E)) .
$$


Here, the map $\tilde{i d}$ is the identity outside a collar of $X$ and compresses the collar slightly so that $X$ fits into $X-\partial X$. In particular, we have for $\pi: X \longrightarrow p t$ the equality

$$
\pi_{!}\left(1_{X}^{M A}\right)=X
$$

Remark 4.1.2. At a glance it seems to be unusual that a manifold $X$ with boundary admits an absolute fundamental class rather than just a relative one. However, observe that the stable normal bundle of its boundary maps to $\partial E$. Hence, the theory $(E, \partial E)$ simply treats $X$ as a manifold without boundary.

Example 4.1.3. Let $E$ be a complex oriented theory and let $E^{\langle n\rangle}$ be the $\langle n\rangle$ spectrum given by the outer $n+1$-fold product of $\left(S^{0} \longrightarrow E\right)$ as in (2.1.1). Then the orientation $g: M U^{\langle n\rangle} \longrightarrow E^{\langle n\rangle}$ defines a complex genus

$$
g_{*}: \Omega_{*}^{(U, f r)^{n}} \cong \pi_{*}\left(M U^{\langle n\rangle}, \partial M U^{\langle n\rangle}\right) \longrightarrow \pi_{*}\left(E^{\langle n\rangle}, \partial E^{\langle n\rangle}\right) .
$$

We would like to compute rationalized complex genera in terms of ordinary singular cohomology. For that, recall from Qui71 that any complex oriented theory $E$ comes with a formal group law $\hat{F}_{E}$ which describes the behaviour of the Euler class for a tensor product of complex line bundles $l_{1}$ and $l_{2}$ :

$$
e_{E}\left(l_{1} \otimes l_{2}\right)=\hat{F}_{E}\left(e_{E}\left(l_{1}\right), e_{E}\left(l_{2}\right)\right) .
$$

Rationally, a formal group law admits a unique strict isomorphism $\exp _{E}(x)$ to the additive formal group law $\hat{F}_{a}(x, y)=x+y$. The latter is induced by the standard orientation of rational singular homology $H \mathbb{Q}$ :

$$
g_{a}: M U \longrightarrow H \mathbb{Q} \longrightarrow H \mathbb{Q} \wedge E \cong S \mathbb{Q} \wedge E
$$

For $j=0,1, \ldots, n-1$ let $\left(x_{i}^{(j)}\right)$ be the system of formal Chern roots in the stable decomposition

$$
T X \cong T X^{(0)} \oplus T X^{(1)} \oplus \cdots \oplus T X^{(n-1)}
$$

of the $(U, f r)^{n}$-manifold $X$. Then the power series $Q_{E}(x)=x / \exp _{E}(x)$ generates a sequence of Chern classes

$$
K_{E}(T X) \stackrel{\text { def }}{=} \bigotimes_{j=0}^{n-1}\left(Q_{E}\left(x_{1}^{(j)}\right) Q_{E}\left(x_{2}^{(j)}\right) \cdots Q_{E}\left(x_{k}^{(j)}\right)\right)
$$

which lies in $\bigotimes_{j=0}^{n-1} H^{*}\left(X ; \pi_{*} E \otimes \mathbb{Q}\right) \cong H^{*}\left(X ; \pi_{*} \bigwedge_{j=0}^{n-1} E \otimes \mathbb{Q}\right)$. Moreover, when setting

$$
\tilde{K}_{E}(T X) \stackrel{\text { def }}{=} \bigotimes_{j=0}^{n-1}\left(\left(Q_{E}\left(x_{1}^{(j)}\right)-1\right)\left(Q_{E}\left(x_{2}^{(j)}\right)-1\right) \cdots\left(Q_{E}\left(x_{k}^{(j)}\right)-1\right)\right)
$$

we even obtain a relative cohomology class which coincides with the absolute one when viewed as an element of $H^{*}\left(X ; \pi_{*}\left(E^{\langle n\rangle}, \partial E^{\langle n\rangle}\right) \otimes \mathbb{Q}\right)$.

Proposition 4.1.4. In $\pi_{*}\left(E^{\langle n\rangle}, \partial E^{\langle n\rangle}\right) \otimes \mathbb{Q}$ we have the formula

$$
g_{*}(X)=\left\langle K_{E}(T X),[X]_{g_{a}^{\langle n\rangle}}\right\rangle=\left\langle\tilde{K}_{E}(T X),[X, \partial X]\right\rangle .
$$


Proof. It is enough to consider the universal case $E=M U$. Since the map

$$
\alpha: \Omega_{*}^{U^{n}} \longrightarrow \Omega_{*}^{(U, f r)^{n}}
$$

is a rational surjection, we can find a closed compact $U^{n}$-manifold $\hat{X}$ with the property $\alpha(\hat{X})=N X$ for some natural number $N$. It is well known how to compute the genus of closed manifolds: the Riemann-Roch formula (see [Dye69]) shows that

$$
\alpha(\hat{X})=\alpha\left(\left\langle K_{M U}(T \hat{X}),[\hat{X}]\right\rangle\right)=\left\langle\tilde{K}_{M U}(T \hat{X}),[\hat{X}]\right\rangle .
$$

Hence, it suffices to establish the equality

$$
\left\langle\tilde{K}_{M U}(T \hat{X}),[\hat{X}]\right\rangle=N\left\langle\tilde{K}_{M U}(T X),[X, \partial X]\right\rangle .
$$

Let $W$ be a $(U, f r)^{n}$-bordism between $\hat{X}$ and $N X$ as pictured for $n=N=$ 1 in Figure 2. Then the boundary of $W$ consists of the manifold $\hat{X}$ and a component $Y$. The stable tangent bundle of $Y$ is the union of $N$ copies of $-T X$ and a part which is classified by $\partial(E U \longrightarrow B U)^{n}$. Hence, we compute with Stokes's theorem:

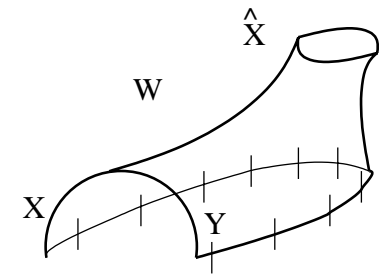

FiguRE 2 .

$$
\begin{aligned}
& \left\langle\tilde{K}_{M U}(T \hat{X}),[\hat{X}]\right\rangle-N\left\langle\hat{K}_{M U}(T X),[X, \partial X]\right\rangle \\
& \quad=\left\langle\tilde{K}_{M U}(T \hat{X}),[\hat{X}]\right\rangle+\left\langle\hat{K}_{M U}(T Y),[Y]\right\rangle \\
& \quad=\left\langle\tilde{K}_{M U}(T W)_{\mid \partial W},[\partial W]\right\rangle=\left\langle d \tilde{K}_{M U}(T W),[W]\right\rangle=0 .
\end{aligned}
$$

We will see that sometimes it is convenient not to distinguish between a $(U, f r)^{n}$ manifold $X$ and $X+Y$ if $Y$ has a $(U, f r)^{n-1} \times U$-structure. For this purpose, for any oriented $\langle n\rangle$-spectrum $E$ we define the map $g_{*}^{\mathbb{Q} / Z}$ to be the genus $g_{*}$ with values in the quotient

$$
\pi_{*}^{\mathbb{Q} / Z} E \stackrel{\text { def }}{=} \pi_{*}(E, \partial E) \otimes \mathbb{Q} / k\left(\pi_{*}(Z E, \partial Z E)\right) .
$$

The functor $Z$ and the map $k$ were defined in the paragraph before 3.2 .5 .

Example 4.1.5. Let $g_{*}: \Omega_{*}^{(U, f r)} \longrightarrow K^{\langle 1\rangle}$ be the genus which comes from the complex orientation of $K$-theory as in 4.1.3. Then for positive even $n$ the map

$$
g_{*}^{\mathbb{Q} / Z}: \Omega_{n}^{(U, f r)} \longrightarrow \pi_{n}^{\mathbb{Q} / Z}\left(K^{\langle 1\rangle}\right) \cong \mathbb{Q} / \mathbb{Z}
$$

sends a $(U, f r)$-manifold to its Todd genus by 4.1.4. Taking values in $\mathbb{Q} / Z$ has the advantage that the composite

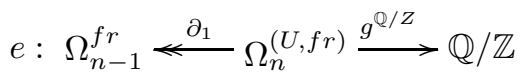

is well defined: by 3.2.5 any two lifts of a framed manifold only differ by a closed $U$ manifold with integral Todd genus. It is well known [CF66] that this map coincides with the Adams $e$-invariant. 
We are going to show that for complex genera the image of $g_{*}^{\mathbb{Q} / Z}$ already takes values in the Adams-Novikov $E_{2}$-term for the sphere based on the complex oriented theory $E$

$$
E_{2}[E] \cong \operatorname{Ext}_{E_{*} E}\left(\pi_{*} E, \pi_{*} E\right) \cong H^{*}\left(\pi_{*} E, E_{*} E\right) .
$$

Let us assume that $1 \in \pi_{0} E$ is not a torsion element. Then $E$ admits a map $r: E \longrightarrow H \mathbb{Q}$ with $r_{*}(1)=1$, and we have

Proposition 4.1.6. There is a factorization

$$
g_{*}^{\mathbb{Q} / Z}: \Omega_{k}^{(U, f r)^{n}} \stackrel{j}{\longrightarrow} E_{2}^{n, k}[M U] \stackrel{E_{2}[g]}{\longrightarrow} E_{2}^{n, k}[E] \stackrel{r \wedge 1}{\longrightarrow} \pi_{k}^{\mathbb{Q} / Z}\left(E^{\langle n\rangle}\right) .
$$

If $E$ is flat and has a torsion free coefficient ring, then the last map $r \wedge 1$ is injective.

Proof. Recall from [Lau99], 3.1, that the group of cycles $C \subset \Omega_{*}^{Z(U, f r)^{n}}$ of $E_{1}[M U]$ makes the diagram

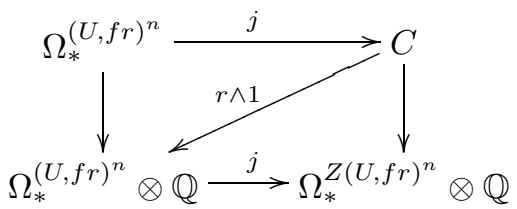

commutative. Thus the desired factorization follows from the naturality of the Adams-Novikov spectral sequence. Moreover, the additional conditions on the theory $E$ ensure that the right vertical and hence the diagonal arrow stay injective when $M U$ is replaced by $E$.

For later purpose we mention

Lemma 4.1.7. Let $t$ be the endofunctor of $\underline{2} \times \underline{2}$ which twists the two factors. Then for any even dimensional $(U, f r)^{2}$-manifold $X$ we have

$$
g_{*}^{\mathbb{Q} / Z}(X)=-g_{*}^{\mathbb{Q} / Z}\left(t^{*} X\right) .
$$

Proof. The manifolds $X$ and $-t^{*} X$ have the same framed corner. Hence, by 3.2.5 the boundaries $\partial_{1} X$ and $-\partial_{1} t^{*} X$ can only differ by an odd dimensional closed $U$ manifold which bounds. Thus, again by 3.2.5 the manifolds $X$ and $-t^{*} X$ coincide up to a $Z(U, f r)^{2}$-manifold which vanishes in the group $\pi_{*}^{\mathbb{Q} / Z} E^{\langle 2\rangle}$.

4.2. Chromatic names of framed Lie groups and the Chern numbers which determine $\beta$. In the last paragraph we have laid the foundation for investigating the chromatic status of framed manifolds. Now we are able to identify a left invariantly framed Lie group $G$ with the corresponding element in the $E_{2}$-term of the Adams-Novikov spectral sequence based on the complex oriented theory $E$.

In 2.1 .2 and 3.2 .3 we regarded $G$ as principal torus bundle over $G / \mathbb{T}$. The cohomology of $G / \mathbb{T}$ is well known $[\mathrm{BH} 58]$ :

$$
H^{*}(G / \mathbb{T}, \mathbb{Q})=H^{*}(B \mathbb{T}, \mathbb{Q}) / \tilde{H}^{*}(B \mathbb{T}, \mathbb{Q})^{W(G)},
$$

and the equation

$$
\left\langle\alpha_{1} \cdot \ldots \cdot \alpha_{m},[G / \mathbb{T}]\right\rangle=|W(G)|
$$

holds. Here, $\alpha_{1}, \ldots, \alpha_{m}$ are the positive roots and $W(G)$ is the Weyl group of $G$. For $j=0, \ldots, r-1$ let $x_{j}$ be the first Chern class of the complex line bundle 
$l_{j}=G \times_{\mathbb{T}} \mathbb{C}$, where $\mathbb{T} \cong\left(S^{1}\right)^{r}$ acts on $\mathbb{C}$ with its $j$ th factor as in 3.2.3. Then the polynomial

$$
\left.\tilde{K}_{E}\left(G \times_{\mathbb{T}} \mathbb{C}^{r}\right)\right) / e \stackrel{\text { def }}{=} \bigotimes_{j=0}^{r-1}\left(Q_{E}\left(x_{j}\right)-1\right) / x_{j}
$$

defines an element in the cohomology ring of $G / \mathbb{T}$ with coefficients in the range of

$$
\pi * E \otimes \cdots \otimes \pi_{*} E \otimes \mathbb{Q} \cong \pi_{*}\left(\bigwedge^{r} E\right) \otimes \mathbb{Q} \longrightarrow \pi_{*}\left(E^{\langle n\rangle}, \partial E^{\langle r\rangle}\right) \otimes \mathbb{Q} .
$$

Corollary 4.2.1. With the above notation we have

$$
g_{*}\left(G \times_{\mathbb{T}}\left(D^{2}\right)^{r}\right)=\left\langle\tilde{K}_{E}\left(G \times_{\mathbb{T}} \mathbb{C}^{r}\right) / e,[G / \mathbb{T}]\right\rangle .
$$

Proof. Write $X$ for the $\langle r\rangle$-manifold $G \times_{\mathbb{T}}\left(D^{2}\right)^{r}$. Let $s: G / \mathbb{T} \longrightarrow X$ be the zero section and let $x^{(j)}$ be the Chern roots of $T X$. By (3.2.2) we know that the tangent bundle of $X$ splits into $p^{*}\left(G \times_{\mathbb{T}} \mathbb{C}^{r}\right)$ and a part which is trivializable. Hence we have $x^{(j)}=p^{*} x_{j}$ and

$$
\tilde{K}_{E}(T X)=p^{*}\left(\tilde{K}_{E}\left(G \times_{\mathbb{T}} \mathbb{C}^{r}\right)\right) .
$$

Moreover, the pair $(X, \partial X)$ defines the Thom space of $l_{0} \oplus \cdots \oplus l_{r-1}$. Let $\tau$ be its Thom class. The element $\rho=\tilde{K}_{E}\left(G \times_{\mathbb{T}} \mathbb{C}^{r}\right) / e$ tries to be the inverse of $\tilde{K}_{E}(T X)$ under the Thom isomorphism:

$$
s^{*}\left(p^{*}(\rho) \cup \tau\right)=\rho \cup e=s^{*} \tilde{K}_{E}(T X) .
$$

In fact, when taking coefficients in $\pi_{*}\left(E^{\langle r\rangle}, \partial E^{\langle r\rangle}\right) \otimes \mathbb{Q}$ we know from 4.1.4 that the fundamental class $[X, \partial X]$ becomes the absolute class $[X]_{g_{a}^{\langle r\rangle}}$, and hence the last equality yields

$$
\left\langle\tilde{K}_{E}(T X),[X, \partial X]\right\rangle=\left\langle p^{*}(\rho) \cup \tau,[X, \partial X]\right\rangle .
$$

Now compute

$$
\begin{aligned}
g\left(G \times_{\mathbb{T}}\left(D^{2}\right)^{r}\right) & =\left\langle\tilde{K}_{E}(T X),[X, \partial X]\right\rangle=\left\langle p^{*}(\rho) \cup \tau,[X, \partial X]\right\rangle \\
& =\left\langle p^{*}(\rho),[X, \partial X] \cap \tau\right\rangle=\left\langle p^{*}(\rho), s_{*}[G / \mathbb{T}]\right\rangle=\langle\rho,[G / \mathbb{T}]\rangle \\
& =\left\langle\tilde{K}_{E}\left(G \times \times_{\mathbb{T}} \mathbb{C}^{r}\right) / e,[G / \mathbb{T}]\right\rangle .
\end{aligned}
$$

We are now well equipped to track down $G$ in the Adams-Novikov spectral sequence. It is convenient to work locally at a prime and set $E=B P$, since $B P$ captures all necessary information and there are better formulae for its exponential.

Example 4.2.2. We check that $S p(2)$ is $\beta$ at the prime 3 [Kna78]. The Weyl group of $S p(2)$ is the wreath product of $\mathbb{Z} / 2$ with the symmetric group $S(2)$ and operates on the $x_{i}$ by permutations and change of signs. Hence, only the products $x_{1} x_{2}^{3}$ and $x_{2} x_{1}^{3}$ do not vanish, and they differ by a sign in $H^{8}(S p(2) / \mathbb{T}, \mathbb{Q})$. Since the positive roots are $x_{1}+x_{2}, x_{1}-x_{2}, 2 x_{1}, 2 x_{2}$, we see that $x_{1}^{3} x_{2}$ is dual to $[S p(2) / \mathbb{T}]$. The $B P$-exponential takes the form (see [Rav86], appendix 2)

$$
\exp (x)=x-m_{1} x^{3}+3 m_{1}^{2} x^{5} \bmod \left(x^{6}\right) .
$$

Using the first $B P$-law $3 m_{1}=v_{1}$, we obtain

$$
(Q(x)-1) / x=v_{1} x / 3-2 v_{1}^{2} x^{3} / 9 \bmod \left(x^{4}\right),
$$


and thus

$$
g_{*}\left(S p(2) \times_{\mathbb{T}}\left(D^{2} \times D^{2}\right)\right)=-2 / 27\left(v_{1} \otimes v_{1}^{2}-v_{1}^{2} \otimes v_{1}\right) .
$$

It suffices to show that this element does not vanish in

$$
\pi_{12}^{\mathbb{Q} / Z} B P^{\langle 2\rangle} \cong \frac{\mathbb{Q}\left\langle v_{1} \otimes v_{1}^{2}, v_{1}^{2} \otimes v_{1}, v_{1}^{3} \otimes 1,1 \otimes v_{1}^{3}\right\rangle}{B P_{12} B P+\mathbb{Q}\left\langle v_{1}^{3} \otimes 1,1 \otimes v_{1}^{3}\right\rangle} .
$$

Recall from Rav86 that $B P_{*} B P$ is a polynomial algebra in generators $t_{1}, t_{2}, \ldots$ and that there is the second $B P$-law $1 \otimes v_{1}=\eta_{R}\left(v_{1}\right)=v_{1}+3 t_{1}$. In particular, when adding $2\left(1 \otimes v_{1}\right)^{3} / 81=2\left(v_{1}^{3} / 81+v_{1}^{2} t_{1} / 9+v_{1} t_{1}^{2} / 3+t_{1}^{3} / 3\right)$ to

$$
-2 / 27\left(v_{1} \otimes v_{1}^{2}-v_{1}^{2} \otimes v_{1}\right)=-2\left(t_{1}^{2} v_{1} / 3+t_{1} v_{1}^{2} / 9\right)
$$

we have

$$
g_{*}^{\mathbb{Q} / Z}\left(S p(2) \times_{\mathbb{T}}\left(D^{2} \times D^{2}\right)\right)=2 t_{1}^{3} / 3 \in \pi_{12}^{\mathbb{Q} / Z} B P^{\langle 2\rangle} .
$$

Next we observe with 4.1.7 that any element in the image of $g_{*}^{\mathbb{Q} / Z}$ is a multiple of $v_{1} \otimes v_{1}^{2}-v_{1}^{2} \otimes v_{1}$ and hence of $t_{1}^{3}$. Moreover, it is easy to see that the map

$$
\kappa: \operatorname{im}\left(g_{*}^{\mathbb{Q} / Z}\right) \longrightarrow \mathbb{Q} / \mathbb{Z}_{(3)} \cong \mathbb{Z}_{3^{\infty}} \quad ; \quad a t_{1}^{3} \mapsto a
$$

is well defined. Thus we may conclude with 4.1.6 that $S p(2)$ has the order 3 and coincides with $\beta$ up to a sign.

Remark 4.2.3. There is an alternative way to argue here: we can use the commutative square (4.1.1) and compute the corresponding element in the reduced cobar complex

$$
j\left(-2\left(t_{1}^{2} v_{1} / 3+t_{1} v_{1}^{2} / 9\right)\right)=-2\left(1\left|t_{1}^{2} v_{1} / 3+1\right| t_{1} v_{1}^{2} / 9\right)=-2\left(t_{1}\left|t_{1}^{2}+t_{1}^{2}\right| t_{1}\right)=2 b_{10} .
$$

Then the formula 5.1 .20 of $\operatorname{Rav86}$ ] says that $S p(2)$ and $\beta$ have the same image in $\operatorname{Ext}^{2}\left(B P_{*} / I_{2}\right)$, which in turn means that they must coincide. It is interesting to note that it is always possible to rewrite the invariant $g^{\mathbb{Q} / Z}$ so that it returns an (integral) representative of the framed manifold in the reduced cobar complex.

Example 4.2.4. We next look at the Lie group $S U(3)$. Its Weyl group $S(3)$ consists of 6 elements, and positive roots are $x_{1}-x_{2}, x_{1}-x_{3}, x_{2}-x_{3}$. Hence, we see as above that $x_{1}^{2} x_{2}$ is dual to the fundamental class of $S U(3) / \mathbb{T}$. At the prime $p=2$ the $B P$-exponential takes the form

$$
\exp (x)=x-m_{1} x^{2}+2 m_{1}^{2} x^{3}+\left(-m_{2}-5 m_{1}^{3}\right) x^{4} \quad \bmod \left(x^{5}\right),
$$

and thus

$$
\begin{aligned}
& g\left(S U(3) \times_{\mathbb{T}}\left(D^{2} \times D^{2}\right)\right)=m_{1}^{2} \otimes\left(m_{2}+2 m_{1}^{3}\right)-\left(m_{2}+2 m_{1}^{3}\right) \otimes m_{1}^{2} \\
& \quad=1 / 8\left(v_{1}^{2} \otimes\left(v_{2}+v_{1}^{3}\right)-\left(v_{2}+v_{1}^{3}\right) \otimes v_{1}^{2}\right) \\
& \quad=1 / 8\left(-v_{1}^{4} t_{1}+3 v_{1}^{3} t_{1}^{2}+2 v_{1}^{2} t_{2}-4 v_{1} v_{2} t_{1}-4 v_{2} t_{1}^{2}+4 v_{1}^{2} t_{1}^{3}\right) .
\end{aligned}
$$

To see the order of this element one may add any term of the form

$$
a\left(1 \otimes m_{1}^{5}\right)+b\left(1 \otimes m_{1}^{2} m_{2}\right) .
$$

Choosing $a=2, b=-1$ we see that our invariant takes the value

$$
g_{*}^{\mathbb{Q} / Z}\left(S U(3) \times_{\mathbb{T}}\left(D^{2} \times D^{2}\right)\right)=-1 / 2\left(11 v_{1} t_{1}^{4}+5 v_{1}^{3} t_{1}^{2}\right) \in \pi_{10}^{\mathbb{Q} / Z} B P^{\langle 2\rangle},
$$

and thus is easily seen to have the order 2 . As there is only one element of second filtration in dimension 8 we may conclude that $S U(3)$ represents $\beta_{2}$ at the prime 2. This result was first obtained in [Ste76] and Woo76]. 
Of course, the formula 4.2.1 applies to any Lie group of arbitrary rank, but the calculations get very long and should be done with a computer. A list of semisimple groups of low rank has been given in [Oss82], together with some question marks for Lie groups of rank 4 and higher.

Instead of looking at more examples of Lie groups we answer the question which was proposed in the introduction:

What are the Chern numbers which determine $\beta$ at the prime 3 ?

A $(U, f r)^{2}$-manifold $X$ comes with a splitting $T X^{(1)}+T X^{(2)}$ of its stable tangent bundle. For $i=1,2$ let $c(i)=\left(c(i)_{1}, c(i)_{2}, \ldots\right)$ be the Chern classes of $T X^{(i)}$ and let $f(c(1), c(2))$ be the polynomial

$$
\begin{aligned}
& 1 / 3\left(-c(1)_{1}{ }^{4} c(2)_{1}{ }^{2}-10 c(2)_{2} c(1)_{4}+5 c(2)_{1}{ }^{2} c(1)_{4}-8 c(1)_{2} c(2)_{2} c(1)_{1}{ }^{2}\right. \\
& \left.\quad+4 c(1)_{2} c(1)_{1}{ }^{2} c(2)_{1}{ }^{2}-5 c(1)_{3} c(1)_{1} c(2)_{1}{ }^{2}+10 c(1)_{3} c(2)_{2} c(1)_{1}+2 c(2)_{2} c(1)_{1}{ }^{4}\right) .
\end{aligned}
$$

Then we have the answer to the question:

Corollary 4.2.5. The corner of a 12 dimensional $(U, f r)^{2}$-manifold $X$ is $\pm \beta$ iff the Chern number

$$
\langle f(c(1), c(2))-f(c(2), c(1)),[X, \partial X]\rangle \in \mathbb{Q} / \mathbb{Z}_{(3)} \cong \mathbb{Z}_{3^{\infty}}
$$

has the order 3.

Proof. One readily verifies by hand or with a computer that the terms of dimension 12 in $\tilde{K}_{B P\langle 2\rangle}(T X)$ have the form

$$
2 / 9\left(f^{\prime}(c(1), c(2))\left(v_{1}^{2} \otimes v_{1}\right)+f^{\prime}(c(2), c(1))\left(v_{1} \otimes v_{1}^{2}\right)\right) \bmod \left(1 \otimes v_{1}^{3}, v_{1}^{3} \otimes 1\right) .
$$

Here, the polynomial $f^{\prime}$ coincides with $f$ up to integral multiples of Chern classes. With 4.1.74.1.4 and the calculations made in 4.2.2 we conclude that

$$
g_{*}^{\mathbb{Q} / Z}(X)=\langle f(c(1), c(2))-f(c(2), c(1)),[X, \partial X]\rangle t_{1}^{3} \in \pi_{12} B P^{\langle 2\rangle} .
$$

Hence the result follows from the fact that $\kappa$ in (4.2.1) was well defined.

4.3. Elliptic cohomology and $\langle 2\rangle$-manifolds. In 4.1.5 we saw that the Todd genus of a $(U, f r)$-manifold is integral iff the $e$-invariant of its boundary is integral or, equivalently, iff its boundary is the corner $\partial_{0} \partial_{1} X$ of a $(U, f r)^{2}$-manifold $X$. Since the Todd genus comes from the complex orientation of $K$-theory, it is not sensitive enough to detect the rich algebraic structure of the Adams-Novikov 2-line MRW77. Of course, complex bordism itself or BP-theory can do the job, but they are hard to track, geometrically and algebraically.

Within the last years a new family of complex oriented cohomology theories has entered algebraic topology: these are represented by elliptic spectra and owe their names to the fact that their formal groups come from elliptic curves (see the survey articles Seg87 and [RS95]). For instance, there is a cohomology theory $E l l^{\Gamma}$ attached to the universal curve over the ring of integral modular forms for the congruence subgroup $\Gamma=\Gamma_{1}(N)$ of $S l_{2}(\mathbb{Z})$ ([Bry90], [Fra92], [Lau99]).

For $N \geq 2$ let $\zeta_{N}$ be a primitive $N$ th root of unity. The elliptic genus takes a manifold $X$ to the modular form with $q$-expansion

$$
a(q) \chi_{-\zeta_{N}}\left(X, \bigotimes_{n=1}^{\infty} S_{q^{n}}\left(T^{*} X \otimes \mathbb{C}\right) \otimes \Lambda_{-q^{n} / \zeta_{N}} T X \otimes \Lambda_{-q^{n} \zeta_{N}} T^{*} X\right) \in \mathbb{Z}\left[\zeta_{N}, 1 / N\right] \llbracket q \rrbracket .
$$


Here, $a(q)$ is the normalization factor

$$
a(q)=\prod_{n=1}^{\infty} \frac{\left(1-q^{n}\right)^{2}}{\left(1-\zeta_{N}\right)\left(1-q^{n} / \zeta_{N}\right)\left(1-q^{n} \zeta_{N}\right)}
$$

and $\chi_{y}(X, W)$ is the $\chi_{y}$-genus of $X$ with values in $W$. It was explained in Wit86 and [HBJ92] how to interpret this expression as the $S^{1}$-equivariant index of an operator which acts on the loop space of $X$. However, a good geometric insight into elliptic cohomology is still missing, and so is its relation to index theorems on manifolds with corners. As a first step in this direction we will show here how the elliptic genus can provide interesting invariants for $\langle 2\rangle$-manifolds.

Consider the $M U^{\langle 2\rangle}$ oriented $\langle 2\rangle$-ring spectrum

$$
D^{\Gamma} \stackrel{\text { def }}{=}\left(\begin{array}{c}
S^{0} \longrightarrow K \\
\downarrow_{l l l^{\Gamma} \longrightarrow K \wedge E l l^{\Gamma}}^{\longrightarrow} K
\end{array}\right) .
$$

Its $\mathbb{Q} / Z$-homotopy is concentrated in even degrees and has been identified in [Lau99], 2.3 , with the group of divided congruences

$\pi_{2 n}^{\mathbb{Q} / Z}\left(D^{\Gamma}\right) \cong\left\{\sum f_{i} \mid f_{0}+\sum f_{i}+f_{n}\right.$ expands integrally for some $\left.f_{0}, f_{n}\right\} \otimes \mathbb{Q} / \mathbb{Z}$.

Here, for each $i$ the element $f_{i}$ is a rational modular form of weight $i$.

Observe that any framed manifold of positive even dimension $n$ lies in the second filtration and hence is the corner of a $(U, f r)^{2}$-manifold.

Lemma 4.3.1. The composite

$$
f: \Omega_{n}^{f r}=F^{2, n+2} \longleftarrow \Omega_{n+2}^{(U, f r)^{2}} \stackrel{g^{\mathbb{Q} / Z}}{\longrightarrow} \pi_{n+2}^{\mathbb{Q} / Z}\left(D^{\Gamma}\right)
$$

is well defined.

Proof. By 4.1.6 it is enough to show that

$$
\Omega_{n}^{f r}=F^{2, n+2} \longleftrightarrow \Omega_{n+2}^{(U, f r)^{2}} \stackrel{j}{\longrightarrow} E_{2}^{2, n+2}[M U]
$$

is well defined. The argument is the same as in 4.1.7. suppose there are $\langle 2\rangle$ manifolds $X, X^{\prime}$ with a common framed corner. Then the boundaries $\partial_{1} X, \partial_{1} X^{\prime}$ only differ by a closed $U$-manifold which bounds. Hence the $\langle 2\rangle$-manifolds $X$ and $X^{\prime}$ coincide up to a $Z(U, f r)^{2}$-manifold which vanishes in the $E_{2}$-term.

The next theorem tells us that the invariant $f$ of 4.3 .1 really deserves its name.

Theorem 4.3.2. A framed manifold is the corner of an $(U, f r)^{3}$-manifold iff the $f$-invariant gives an integral inhomegeneous modular form for two levels $\geq 2$ which are relatively prime to each other.

Before giving the proof we need a change of rings result which is parallel to the one of [Bak97] for level 1 elliptic cohomology. Let $F$ be a formal group law over the graded $\mathbb{Z}_{(p)}$-algebra $R_{*}$. For $k=0,1, \ldots$ let $v_{k}$ be the images of the Hazewinkel generators under the map from $B P_{*}$ to $R_{*}$ which classifies the typicalization of $F$. Write $H^{*} R$ for the cohomology

$$
H^{*}\left(R_{*}, \Gamma_{R}\right) \cong \operatorname{Ext}_{\Gamma_{R}}\left(R_{*}, R_{*}\right) ; \Gamma_{R} \stackrel{\text { def }}{=} R_{*} \otimes_{M U_{*}} M U_{*} M U \otimes_{M U_{*}} R_{*} .
$$


Then we have

Lemma 4.3.3. Assume that the ideal $I_{n}=\left(p, v_{1}, \ldots, v_{n-1}\right)$ forms a regular sequence which does not span all of $R$, and let $v_{n}$ be invertible modulo $I_{n}$. Then there is a natural isomorphism between $H^{*}\left(v_{n}^{-1} B P_{*}\right)$ and $H^{*}(R)$.

Proof. We may assume that $F$ is $p$-typical. By [HS96], 3.4, we find a faithfully flat extension $S(k)_{*}$ of $v_{k}^{-1} R_{*} / I_{k}$ for each $0 \leq k \leq n$ with the property that over $S(k)_{*}$ there is an isomorphism of the formal group law $F$ to the Honda formal group of height $k$. Thus Hopkins's change of rings theorem [HS96], 3.3, implies that

$$
H^{*}\left(v_{k}^{-1} R / I_{k}\right) \cong H^{*}(S(k)) \text {. }
$$

Since the $B P_{*}$-module structure of $S(k)_{*}$ factors over $K(k)_{*}=\mathbb{F}_{p}\left[v_{k}, v_{k}^{-1}\right]$ and $S(k)_{*}$ is faithfully flat over $K(k)_{*}$, we may conclude, with the same argument, that

$$
\operatorname{Ext}_{\Gamma_{R}}\left(R_{*}, v_{k}^{-1} R_{*} / I_{k}\right) \cong H^{*}\left(v_{k}^{-1} R / I_{k}\right) \cong H^{*}\left(K(k)_{*}\right)
$$

and thus

$$
\operatorname{Ext}_{\Gamma_{R}}\left(R_{*}, v_{k}^{-1} R_{*} / I_{k}\right) \cong \operatorname{Ext}_{\Gamma_{v_{n}^{-1} B P_{*}}}\left(v_{n}^{-1} B P_{*},\left(v_{k} v_{n}\right)^{-1} B P_{*} / I_{k}\right) .
$$

Next consider the nil $B P_{*} B P$-comodule $v_{s}^{-1} B P_{*} /\left(p^{\infty}, \ldots, v_{s-1}^{\infty}\right)$ for some integer $s$. One verifies (or simply uses [MR77], 3.11, 3.12) that it is a direct limit of comodules each of which admits a finite filtration $\left(F^{r}\right)$ with

$$
F^{r} / F^{r+1} \cong v_{k}^{-1} B P_{*} / I_{k}
$$

for some $k=k(r)$. Hence, when we apply the canonical transformations of exact functors

$$
v_{n}^{-1} B P_{*} \otimes_{B P_{*}}-\longrightarrow v_{n}^{-1} R \otimes_{B P_{*}}-\longleftarrow R \otimes_{B P_{*}}-
$$

and use the obvious five lemma argument in cohomology, we obtain isomorphisms on the corresponding chromatic $E_{2}$-terms [Rav86]:

$$
E_{2}^{*, *, *}\left[v_{n}^{-1} B P_{*}\right] \cong E_{2}^{*, *, *}\left[v_{n}^{-1} R\right] \cong E_{2}^{*, *, *}[R] .
$$

Thus the claim follows from the convergence of the chromatic spectral sequences.

Proof of 4.3.2. By 3.2.5 it suffices to show that

$$
E_{2}^{2, n+2}[M U] \longrightarrow E_{2}^{2, n+2}\left[E l l^{\Gamma}\right] \longrightarrow \pi_{n+2}^{\mathbb{Q} / Z}\left(E l l^{\Gamma^{\langle 2\rangle}}\right) \longrightarrow \pi_{n+2}^{\mathbb{Q} / Z}\left(D^{\Gamma}\right)
$$

is injective after localization at each prime which does not divide the level. Only the first arrow needs to be checked, since the second and the last arrow are injective by 4.1.6 and Lau99, 3.13, respectively. When applying the above lemma to the $\operatorname{ring} R_{*}=E l l_{*}^{\Gamma}$ we may replace elliptic cohomology by $v_{2}^{-1} B P_{*}$. The chromatic $E_{2}$ term of $v_{2}^{-1} B P_{*}$ simply is the truncated one of $B P_{*}$ :

$$
E_{2}^{s, *, *}\left[v_{2}^{-1} B P_{*}\right] \cong \begin{cases}E_{2}^{s, *, *}\left[B P_{*}\right] & \text { for } 0 \leq s \leq 2 \\ 0 & \text { else. }\end{cases}
$$

Since the 0-column is concentrated in bidegree $(0,0)$, all differentials $d_{2}$ and higher which arrive in the $s$-column for $s \leq 2$ vanish. Hence, we obtain the desired injectivity for $r+s=2$ as the composite of

$$
E_{\infty}^{r, s, *}\left[B P_{*}\right] \hookrightarrow E_{2}^{r, s, *}\left[B P_{*}\right]=E_{2}^{r, s, *}\left[v_{2}^{-1} B P_{*}\right]=E_{\infty}^{r, s, *}\left[v_{2}^{-1} B P_{*}\right]
$$




\section{REFERENCES}

[Ada74] J.F. Adams, Stable homotopy and generalized homology, The University of Chicago Press, 1974. MR 53:6534

[AS74] M.F. Atiyah and L. Smith, Compact Lie groups and the stable homotopy of spheres, Topology 13 (1974), 135-142. MR 49:8013

[Bak97] A. Baker, Hecke operations and the Adams e $e_{2}$-term based on elliptic cohomology, Canad. Math. Bull. 42 (1999), 129-138. MR 2000c:55008

[BH58] A. Borel and F. Hirzebruch, Characteristic classes and homogeneous spaces, I-III, Am. J. Math. 80-82 (1958), 458-538, 315-382,491-504. MR 21:1586 MR 22:988 MR 22:11413.

[Bry90] J.L. Brylinski, Representations of loop groups, Dirac operators on loop space, and modular forms, Topology 29 (1990), 461-480. MR 91j:58151

[Cer61] J. Cerf, Topologie de certains espaces de plongements, Bull. Soc. Math. France 89 (1961), 227-380. MR 25:3543

[CF66] P.E. Conner and E.E. Floyd, The relation of cobordism to $K$-theories, LNM 28, Springer, Berlin, 1966. MR 35:7344

[Dol78] A. Dold, Geometric cobordism and the fixed point transfer, Algebraic Topology, Lecture Notes in Mathematics, vol. 673, Springer Verlag, 1978, pp. 32-87. MR 80g:57052

[Dou61] A. Douady, Variétés à bord anguleux et voisinages tubulaires, théorèmes d'isotopie et de recollement, Séminaire Henri Cartan 1961/62, Exposé 1, Secrétariat Math., Paris, 1961/62. MR 28:3435

[Dye69] E. Dyer, Cohomology theories, W.A. Benjamin, New York Amsterdam, 1969. MR 42:3780

[Elm88] A.D. Elmendorf, The Grassmannian geometry of spectra, Journal of Pure and Applied Algebra 54 (1988), 37-94. MR 90c:55007

[Fra92] J. Franke, On the construction of elliptic cohomology, Math. Nachr. 158 (1992), 43-65. MR 94h:55007

[HBJ92] F. Hirzebruch, T. Berger, and R. Jung, Manifolds and modular forms, Aspects of Mathematics, vol. E20, Vieweg, Braunschweig, 1992. MR 94d:57001

[HS96] M. Hovey and H. Sadofsky, Invertible spectra in the E(n)-local stable homotopy category, J. London Math. Soc. (2) 60 (1999), 284-302. CMP 2000:04

[Jän68] K. Jänich, On the classification of $O(n)$-manifolds, Math. Annalen 176 (1968), 53-76. MR 37:2261

[Kna78] K. Knapp, Rank and Adams filtration of a Lie group, Topology 17 (1978), 41-52. MR 57:10703

[Lau99] G. Laures, The topological q-expansion principle, Topology 38 (1999), 387-425. MR 2000c:55009

[Lil73] J. Lillig, A union theorem for cofibrations, Arch. Math. 24 (1973), 410-415. MR 48:12512

[LMS80] L.G. Lewis, J.P. May, and M. Steinberger, Equivariant stable homotopy theory, Lecture Notes in Mathematics, vol. 1213, Springer Verlag, 1986. MR 88e:55002

[MR77] H.R. Miller and D.C. Ravenel, Morava stabilizer algebras and the localization of Novikov's $E_{2}$-term, Duke Math. Journal 44 (1977), 433-447. MR 56:16613

[MRW77] H.R. Miller, D.C. Ravenel, and W.S. Wilson, Periodic phenomena in the AdamsNovikov spectral sequence, Annals of Math. 106 (1977), 469-516. MR 56:16626

[Oss82] E. Ossa, Lie-groups as framed manifolds, Topology 21 (1982), 315-323. MR 83e:55009

[Qui71] D. Quillen, Elementary proofs of some results of cobordism theory using Steenrod operations, Advances in Mathematics 7 (1971), 29-56. MR 44:7566]

[Rav86] D.C. Ravenel, Complex cobordism and stable homotopy groups of spheres, Academic Press, Orlando, 1986. MR 87j:55003

[Ray79] N. Ray, Invariants of reframed manifolds, Proc. London Math. Soc. 39 (1979), 253-275. MR 81a:55024

[RS95] P.S. Landweber D.C. Ravenel and R.E. Stong, Periodic cohomology theories defined by elliptic curves, The Cech Centennial: A Conference on Homotopy Theory (Providence) (M. Cenkl and H. Miller, eds.), Contemp. Math., vol. 181, Amer. Math. Soc., 1995, pp. 317-337. MR 96i:55009

[Seg87] G. Segal, Elliptic cohomology, Séminaire Bourbaki 1987/88, Exposé 695, Astérisque, no. 161-162, Soc. Math. France, Paris, 1988, pp. 187-201. MR 91b:55005 
[Ste76] B. Steer, Orbits and the homotopy class of a compactification of a classical map, Topology 15 (1976), 383-393. MR 56:3864

[Sto68] R. E. Stong, Notes on cobordism theory, Princeton University Press, Princeton, 1968. MR 40:2108

[Str68] A. Strøm, Note on cofibrations. II, Math. Scand. 22 (1968), 130-142. MR 39:4846

[Tho54] R. Thom, Quelques propriétés globales des variétés différentiables, Comment. Math. Helv. 28 (1954), 17-86. MR 15:890a

[Wit86] E. Witten, The index of the Dirac operator in loop space, Elliptic Curves and Modular Forms in Algebraic Topology (Berlin and New York), Lecture Notes in Math., no. 1326, Springer, 1986, pp. 161-181. MR 41a:57021

[Woo76] R.M.W. Wood, Framing the exceptional Lie group $G_{2}$, Topology 15 (1976), 303-320. MR 58:7665

Fachbereich Mathematik, Johannes Gutenberg Universität Mainz, D-55099 Mainz, Germany

Current address: Mathematisches Institut der Universität Heidelberg, Im Neuenheimer Feld 288, D-69120 Heidelberg, Germany

E-mail address: gerd@laures.de 